\title{
Feasibility of Engaging an Integrated Digital Network to Implement Guideline-Directed Exercise Recommendations and Patient Reported Outcomes for Adults With Heart Failure
}

Kelly Bosak ( $\sim$ kbosak@kumc.edu )

University of Kansas Medical Center https://orcid.org/0000-0002-4568-7040

Nadeen Abusalim

University of Kansas Medical Center

\section{Research note}

Keywords: Implementation Science, Feasibility, Guideline-Directed Evaluation and Management (GDEM), exercise, heart failure, Greater Plains Collaborative

Posted Date: May 13th, 2021

DOl: https://doi.org/10.21203/rs.3.rs-505938/v1

License: (c) (1) This work is licensed under a Creative Commons Attribution 4.0 International License.

Read Full License 


\section{Abstract}

Objective

Heart failure (HF) is a chronic condition affecting millions of people worldwide. Exercise is safe and effective for preventing or slowing HF progression. Despite the benefits, implementation of guidelinedirected exercise recommendations for adults with HF is lacking. The objective of this investigation was to assess the feasibility of an established integrated digital network to support a future larger implementation study to improve uptake of guideline-directed exercise recommendations for HF patients.

Results

A distributive query in REDCap was sent from the lead institution, completed, and returned by the information managers at each health system in the network. Descriptive analysis found ten (83\%) of 12 sites responded to the initial query. Findings included adults, age $\geq 18$ years with HF (ICD10: I50) since January 1, $2019 \mathrm{n}=171,715$ (range 4,279-36,856). Two sites (17\%) reported PROMIS measures accessible in the electronic health record (EHR). Six (50\%) reported PROMIS measures accessible outside the EHR. Four (33\%) sites participated in the GWTG-HF registry. This pre-implementation investigation indicated that a future larger study is feasible with this digital network with a large population of HF patients. Feasibility of including patient reported outcomes in a future study may be improved with access to these measures in the EHR.

\section{Contributions To The Literature}

- We report findings from a distributive query across an integrated, digital network of leading health systems in the region to determine feasibility of leveraging this collaborative for a future larger implementation study.

- This paper adds to the understanding of the process of pre-implementation mapping, which combines implementation science and intervention mapping approaches.

- It is essential to understanding that implementation outcome measures are different from clinical treatment or system outcomes, and involve measures of acceptability, adoption, appropriateness, feasibility, and sustainability.

\section{Introduction}

Evidence-based guidelines are recognized as the standard of care to improve outcomes for the 6.5 million people in the United States affected by heart failure (HF). ${ }^{1}$ The current HF guidelines were developed and endorsed by multiple national organizations including the Heart Failure Society of America (HFSA), American College of Cardiology (ACC), and the American Heart Association (AHA) ${ }^{2,3}$ The AHA also launched Get With The Guidelines-Heart Failure (GWTG-HF), a voluntary, hospital-based initiative in 2005. 
This initiative uses a national data registry and benchmarking program to promote guideline adoption. ${ }^{2}$ More recently, guideline-directed management and therapy (GDMT) entered the lexicon to promote optimal therapies for adults with HF based on the current evidence. Medication optimization is a prominent part of GDMT. ${ }^{4}$ Health behaviors have received less attention, namely, exercise to improve HF outcomes.

Multiple large clinical trials support the safety and efficacy of exercise to prevent or slow HF progression, translating to improved health-related quality of life. ${ }^{2}$ Despite the benefits, there is a lack of implementation of guideline-directed exercise recommendations for HF patients. The objective of this preimplementation investigation was to assess the feasibility of engaging the Greater Plains Collaborative, ${ }^{5}$ an integrated digital network of 12 leading health systems across 10 states in the Midwestern United States to support a future implementation study and improve adoption of exercise guideline recommendations for HF patients. The specific aims were 1) Determine the number of patients with a diagnosis of HF treated at each of the 12 leading health systems in the network; and 2) Assess the status of PRO measure accessibility in the EHR systems across the network. The following research questions were also addressed: Q1) Can the information needed from the health systems across the collaborative be effectively accessed and reported? Q2) Is centralized facilitation of the network efficient in extracting data in a timely manner?

\section{Main Text}

Implementation science includes the methods needed for implementation, evaluation, and maintenance of guideline-directed recommendations. ${ }^{6,7}$ The literature indicates that passive approaches to guideline implementation are largely ineffective ${ }^{8}$ as uptake does not occur spontaneously or naturally. ${ }^{9}$ Comprehensive multilevel approaches are more effective ${ }^{10}$ when they involve stakeholder involvement ${ }^{11-}$ 13 and are tailored to a specific audience. ${ }^{14}$ Identification of unique approaches to implementation is needed beginning in the early developmental stages to maximize uptake of guideline-directed therapies and emphasize feasibility and sustainability while minimizing cost and disparities. ${ }^{15,16}$

The uptake of guideline-directed therapies informed by implementation science gives reason to a practice, ${ }^{17}$ and promotes meaningful patient outcomes. ${ }^{18}$ To assist the development of an implementation intervention for exercise in HF patients, implementation mapping followed this preimplementation query. Implementation mapping involved a systematic approach, combining implementation science and intervention mapping to plan intervention techniques and strategies. ${ }^{19}$ Implementation mapping promoted consistent use of concepts and constructs that aligned with the study objectives. The implementation mapping process also helped to identify theory-based behavior change strategies and techniques advantageous for promoting guideline uptake.

Multiple health systems were queried across an existing integrated digital network to determine the feasibility of leveraging this network for a future implementation study. The standard of care for adults with HF is defined by Guideline-Directed Evaluation and Management (GDEM) based on the current state- 
of-the science. A key behavioral factor in the HF guidelines that is often overlooked is exercise. Adults with HF experience poorer quality of life outcomes compared to patients with other chronic conditions attributed to symptoms of dyspnea, fatigue, and limitations in exercise tolerance. ${ }^{20}$ Exercise is safe and effective for adults with HF, a recommendation supported by the highest level of evidence. ${ }^{2}$ Implementation science is key to accelerating the uptake of this guideline recommendation. This project explored the potential to engage the integrated digital network to accelerate the implementation of guideline-directed exercise in adults with HF.

The integrated digital network or collaborative is an established infrastructure led by our University health system, a regional referral center for adults with HF in the Midwest. The collaborative represents a diverse set of patients and institutions ranging from cutting-edge academic medical centers to local community health clinics. These connections allow research to be conducted and have expanded to allow the infrastructure necessary for multi-site research. The health systems throughout the network comprise 430 clinics, 1,800 primary care providers, and 7,600 specialist providers and continue to grow. ${ }^{21}$ This infrastructure contains data for specialty populations, such as adults with HF to contribute to the creation of new knowledge. The sites have teams with extensive expertise with electronic health record (EHR) systems and terminology standardization.

The model or structure for the data used across the collaborative is referred to as the Common Data Model (CDM). Standardization is necessary across systems for data sharing and to be useful in research. The network health systems incorporate the CDM for electronic health record and billing data. ${ }^{22} \mathrm{~A}$ field is included in the CDM that contains a patient reported outcomes measure. While these measures are included in the CDM, the degree of accessibility and ease of use in the EHRs at each health system across the collaborative was unknown. This information was needed for future implementation research primarily to scale and sustain an implementation intervention.

Patient reported outcomes traditionally included symptoms routinely assessed during clinic encounters with HF patients. The defining clinical symptoms of HF are dyspnea, fatigue, and limitations in exercise tolerance; however, the experience of patients with HF extends beyond these symptoms to include a range of physical, mental, and social effects. ${ }^{23}$ Assessment is generally limited to standard measures of biological or physiological symptoms to identify condition deterioration in $\mathrm{HF}^{24}$

There is growing recognition of the need for evaluation of HF symptoms using validated, standardized measures from the patient's perspective, such as the Patient Reported Outcome Measures Information System (PROMIS). ${ }^{25}$ The PROMIS measures include general health items and multiple condition specific subscales to assess physical, mental, and social health, and overall health-related quality of life. Without consideration of the patient's perspective, the role of the patient's physical, mental and social health and attitudinal factors in HF may go unrecognized. ${ }^{26}$ The degree of integration of patient reported outcomes in the electronic health record (EHR) systems across the digital network was unknown. 


\section{Materials And Methods}

The purpose of this study was to assess the feasibility of including multiple health systems across an integrated digital network in a future implementation intervention to disseminate and implement guideline-directed exercise recommendations for adults with HF. The data available through the EHR systems of the network of 12 leading health systems across 10 states in the Midwestern US was queried using a distributive query in REDCap.

The study design was a descriptive distributive query across the existing digital network. The query was sent from the lead institution to the information managers at each participating institution using a REDCap survey for completion and was returned electronically for analysis (see Table 1). Our institution leads the collaborative and was the central coordinating site where the query was distributed. Centralized facilitation of this integrated digital network was intended to allow for greater efficiency in data extraction from these real-world clinical settings. The network recognizes one primary Institutional Review Board (IRB) for research across multiple sites. For this investigation, our University IRB served in this capacity and granted approval for this investigation. This centralized approach was also intended to facilitate extraction of deidentified data in a timely manner. This information was needed to determine the feasibility of using this network for a future implementation study.

\section{Results}

The size of each health system and the total number of HF patients treated at each institution in the network varied. The total number of patients receiving care at collaborative institution(s) in each state ranged from just under 500,000 to more than $2,500,000.5,22$ All adults with $\mathrm{HF}$ with at least one encounter in the past 5 years were included in the analysis. Ten (83\%) of 12 sites responded to our REDCap survey (Table 2) on the initial request. The patients with HF (ICD10: I50) since January 1, 2019 for adults, age $\geq 18$ years was $n=171,715$ (range $4,279-36,856$ ). One site added the comment that in a recent query of their database, over 50 variations of the ICD10 code 150 for HF were identified. All ICD10 150 codes were included in this analysis.

Only two (25\%) of 8 sites reported patient reported outcome measures used in routine clinical practice. PROMIS measures are the only measures currently included in the CDM, and two sites across the collaborative had access to PROMIS measures in their EHRs. Six (75\%) of 8 sites reported PROMIS measures accessible outside of their EHR. One of the sites reported a total of 587 completed PROMIS surveys outside of the EHR from an unidentified ambulatory clinic that did not involve HF patients. One site responding to the PRO question added a text response with the comment, "The yes response re: PRO data outside the CDM is dependent on our site using the instruments of interest, so it's a cautious yes." Also, of note, we identified participation in the American Heart Association's national GWTG-HF registry across the network, although this was not included in the REDCap query to the information managers. Four $(33 \%)$ of the sites were found to be participating to varying degrees in the registry. This information 
contributed to the identification of determinants of exercise guideline implementation for a future larger implementation study across the collaborative.

\section{Discussion}

A large population of adults with a diagnosis of HF was identified across the collaborative. Efficient centralized facilitation for extracting the requested data was accomplished in a timely manner, supporting the feasibility of using this vast network for a future larger implementation study. A follow-up query was not sent to the non-responders but is likely to have resulted in $100 \%$ response rate across sites and will be used in future investigations. It is the nature of the collaborative to support data sharing across sites, however, no previous implementation studies were reported that used this network. The majority of sites were not participating in the GWTG-HF national registry. The reasons for lack of participation are unknown. Financial barriers may be a factor due to the costs to the institution for registry participation.

Few health systems in the network collected PRO measures within their EHR. This is not optimal for ease of access for routine use. Over half of the health systems reported accessibility of PRO measures outside of their EHR, but this involved accessing the institutions data warehouse, which required additional time and resources. There is growing interest in integrating PROs into EHRs to improve usability. A PRO field exists in the Common Data Model (CDM) to standardize data across the network. This is the initial step toward improving the routine use of PROs for clinical benchmarking and research. Our research provides support for populating the most widely used measures, like PROMIS in the CDM across the collaborative.

\section{Conclusions}

In conclusion, this pre-implementation project confirmed the feasibility of engaging health systems across an integrated digital network in the region to scale and sustain exercise guideline implementation. The collaboration between investigators and the data managers at the various healthcare systems was effective. The digital connections of the collaborative enable centralized facilitation across clinics and health systems, with considerable potential to improve dissemination, implementation, scalability, and sustainability. Including patient reported outcomes measures (PROs) in the EHRs across the network will improve ease of access and thus, use of these measures. Exercise is essential for the large and growing HF population. Ultimately, this research will positively impact implementation of exercise recommendations allowing adults with HF to live longer, more fulfilling lives.

\section{Limitations}

This small feasibility query was conducted as part of the pre-implementation process preceding our implementation mapping project and informing a future larger investigation. The existing integrated digital network across leading health systems in the Midwest was queried, and it was determined to be 
feasible for a future, larger implementation trial. We acknowledge that this network is unique, and thus, findings are not intended for generalization to other digital infrastructures.

\section{Declarations}

\section{Ethics Approval and Consent to Participate}

This investigation was approved by the Institutional Review Board at the University of Kansas Medical Center as an Exempt study, since no human subjects were involved in this query and no unique patient identifiers were included in the data obtained from the distributive query.

\section{Consent for Publication}

Not Applicable

\section{Availability of Data and Materials}

The data generated from the distributive query during the current study are available in a data file stored on the institution's secure server, accessible only by password protection.

\section{Competing Interests}

The authors declare that they do not have any financial or non-financial competing interests.

\section{Funding}

No funding was received for this investigation.

\section{Authors' Contributions}

Dr. Bosak developed the idea for this feasibility query, lead the development of the REDCap query for use across the integrated, digital network, received responses from the participating health systems and analyzed the data, and drafted the manuscript.

Ms. Abusalim assisted with reviewing the responses from the health systems across the collaborative, assisted with data analysis, revised, and edited the manuscript. She also presented a poster of the findings at local and regional research meetings.

\section{Acknowledgements}

We would like to acknowledge the generous contributions of Medical Informatics Coordinators from the Center for Medical Informatics \& Enterprise Analytics at the University of Kansas Medical Center. This team assisted with writing the data query for distribution across the digital network. 
REDCap at University of Kansas Medical Center is supported by CTSA grant from NCRR and NCATS awarded to the University of Kansas Medical Center for Frontiers: University of Kansas Clinical and Translational Science Institute.

\section{References}

1. Benjamin EJ, Muntner P, Alonso A, et al. Heart Disease and Stroke Statistics-2019 Update: A Report From the American Heart Association. Circulation. 2019;139(10):e56-e528.

2. Yancy CW, Jessup M, Bozkurt B, et al. 2017 ACC/AHA/HFSA Focused Update of the 2013 ACCF/AHA Guideline for the Management of Heart Failure: A Report of the American College of Cardiology/American Heart Association Task Force on Clinical Practice Guidelines and the Heart Failure Society of America. Circulation. 2017;136(6):e137-e161.

3. Lindenfeld J, Albert NM, Boehmer JP, et al. HFSA 2010 Comprehensive Heart Failure Practice Guideline. Journal of cardiac failure. 2010;16(6):e1-194.

4. Yancy CW, Jessup M, Bozkurt B, et al. 2016 ACC/AHA/HFSA Focused Update on New Pharmacological Therapy for Heart Failure: An Update of the 2013 ACCF/AHA Guideline for the Management of Heart Failure: A Report of the American College of Cardiology/American Heart Association Task Force on Clinical Practice Guidelines and the Heart Failure Society of America. Journal of the American College of Cardiology. 2016;68(13):1476-1488.

5. Waitman LR, Aaronson LS, Nadkarni PM, Connolly DW, Campbell JR. The Greater Plains Collaborative: a PCORnet Clinical Research Data Network. J Am Med Inform Assoc. 2014;21(4):637641.

6. Eccles MP, Mittman BS. Welcome to Implementation Science. Implementation Science. 2006;1(1):1.

7. Powell BJ, Waltz TJ, Chinman MJ, et al. A refined compilation of implementation strategies: results from the Expert Recommendations for Implementing Change (ERIC) project. Implement Sci. 2015;10:21.

8. Bero LA, Grilli R, Grimshaw JM, Harvey E, Oxman AD, Thomson MA. Closing the gap between research and practice: an overview of systematic reviews of interventions to promote the implementation of research findings. The Cochrane Effective Practice and Organization of Care Review Group. Bmj. 1998;317(7156):465-468.

9. Glasgow RE, Klesges LM, Dzewaltowski DA, Bull SS, Estabrooks P. The future of health behavior change research: what is needed to improve translation of research into health promotion practice? Annals of behavioral medicine : a publication of the Society of Behavioral Medicine. 2004;27(1):3-12.

10. Richard L, Gauvin L, Raine K. Ecological models revisited: their uses and evolution in health promotion over two decades. Annu Rev Public Health. 2011;32:307-326.

11. Green LW. Making research relevant: if it is an evidence-based practice, where's the practice-based evidence? Fam Pract. 2008;25 Suppl 1:i20-24. 
12. Glasgow RE, Goldstein MG, Ockene JK, Pronk NP. Translating what we have learned into practice. Principles and hypotheses for interventions addressing multiple behaviors in primary care. Am J Prev Med. 2004;27(2 Suppl):88-101.

13. Wandersman A, Duffy J, Flaspohler P, et al. Bridging the gap between prevention research and practice: the interactive systems framework for dissemination and implementation. $A m \mathrm{~J}$ Community Psychol. 2008;41(3-4):171-181.

14. Lomas J. Diffusion, dissemination, and implementation: who should do what? Ann N Y Acad Sci. 1993;703:226-235; discussion 235-227.

15. Glasgow RE, Vinson C, Chambers D, Khoury MJ, Kaplan RM, Hunter C. National Institutes of Health approaches to dissemination and implementation science: current and future directions. Am J Public Health. 2012;102(7):1274-1281.

16. Brownson RC, Jacobs JA, Tabak RG, Hoehner CM, Stamatakis KA. Designing for dissemination among public health researchers: findings from a national survey in the United States. Am J Public Health. 2013;103(9):1693-1699.

17. Davidoff F, Dixon-Woods M, Leviton L, Michie S. Demystifying theory and its use in improvement. BMJ Qual Saf. 2015;24(3):228-238.

18. Damschroder LJ, Aron DC, Keith RE, Kirsh SR, Alexander JA, Lowery JC. Fostering implementation of health services research findings into practice: a consolidated framework for advancing implementation science. Implement Sci. 2009;4:50.

19. Fernandez ME, Ten Hoor GA, van Lieshout S, et al. Implementation Mapping: Using Intervention Mapping to Develop Implementation Strategies. Front Public Health. 2019;7:158.

20. Flynn KE, Lin L, Ellis SJ, et al. Outcomes, health policy, and managed care: relationships between patient-reported outcome measures and clinical measures in outpatients with heart failure. American heart journal. 2009;158(4 Suppl):S64-71.

21. He J, Hu Y, Zhang X, Wu L, Waitman LR, Liu M. Multi-perspective predictive modeling for acute kidney injury in general hospital populations using electronic medical records. JAMIA Open. 2019;2(1):115122.

22. Waitman LR. The greater plains collaborative (GPC): Building informatics capacity for clinical/translational and pragmatic research. 2016. www.kumc.edu/kumcri/research-trainingand.../2016-lectures.html. Published February 17, 2016.

23. Ahmad FS, Kallen MA, Schifferdecker KE, et al. Development and Initial Validation of the PROMISPlus-HF Profile Measure. Circulation: Heart Failure. 2019;12(6):e005751.

24. DeVon HA, Vuckovic K, Ryan CJ, et al. Systematic review of symptom clusters in cardiovascular disease. European journal of cardiovascular nursing : journal of the Working Group on Cardiovascular Nursing of the European Society of Cardiology. 2017;16(1):6-17.

25. Cella D, Riley W, Stone A, et al. The Patient-Reported Outcomes Measurement Information System (PROMIS) developed and tested its first wave of adult self-reported health outcome item banks: 2005-2008. Journal of clinical epidemiology. 2010;63(11):1179-1194. 
26. Chien HC, Chen HM, Garet M, Wang RH. Predictors of physical activity in patients with heart failure: a questionnaire study. The Journal of cardiovascular nursing. 2014;29(4):324-331.

\section{Tables}

Table 1 is not available with this version.

Table 2. Feasibility Data Table

\begin{tabular}{|c|c|c|c|c|}
\hline $\begin{array}{l}\text { Health } \\
\text { System }\end{array}$ & $\begin{array}{l}\text { Count Adults } \\
\text { (>=18 yr) } \\
\text { with HF } \\
\text { (ICD10: 150) } \\
\text { Since } \\
1 / 1 / 2019\end{array}$ & $\begin{array}{l}\text { PRO-CM } \\
\text { CDM Table } \\
\text { in EHR? } \\
(y / n)\end{array}$ & $\begin{array}{l}\text { Able to } \\
\text { Collect PRO } \\
\text { measures } \\
\text { outside } \\
\text { CDM? }(y / n)\end{array}$ & \begin{tabular}{|l} 
GWTG-HF \\
Registry? \\
(y/n)
\end{tabular} \\
\hline $\begin{array}{l}10 / 12 \\
\text { responses }\end{array}$ & $\begin{array}{l}\text { Total } \\
n=171,715 \\
\text { (range } \\
4,297- \\
36,536 \text { ) }\end{array}$ & $\begin{array}{l}\mathrm{Y}=2 \\
\mathrm{~N}=8 \\
\mathrm{NR}=2 \\
\text { (PRO-CM } \\
\text { CDM table } \\
\text { populated?) }\end{array}$ & $\begin{array}{l}Y=6 \\
N=2 \\
N R=2\end{array}$ & $\begin{array}{l}Y=3 \\
N=7 \\
N R=2\end{array}$ \\
\hline KS & 10,820 & No & Yes & Y \\
\hline $\mathrm{MN}(1)$ & 36,856 & Yes & NA & $\mathrm{N}$ \\
\hline$\overline{M N}(2)$ & 9,616 & No & No & Y \\
\hline WI & 15,662 & No & Yes & $\mathrm{N}$ \\
\hline$\overline{\mathrm{NE}}$ & 7,768 & Yes & NA & $\mathrm{N}$ \\
\hline$\overline{\mathrm{MO}}$ & 4,297 & No & Yes & $\mathrm{N}$ \\
\hline$\overline{T X(1)}$ & 15,099 & No & Yes & $\mathrm{N}$ \\
\hline UT (1) & 28,842 & No & Yes & Y \\
\hline IIN & 36,536 & No & No & $\mathrm{N}$ \\
\hline $\mid \mathrm{IA}$ & 6,219 & No & Yes & $\mathrm{N}$ \\
\hline
\end{tabular}

